\title{
Pricing corporate bonds with dynamic default barriers
}

\author{
Cho-Hoi Hui \\ Banking Policy Department, Hong Kong Monetary Authority, 30th Floor, 3, Garden Road, Hong \\ Kong, and Department of Economics and Finance, City University of Hong Kong
}

\section{Chi-Fai Lo}

Physics Department, The Chinese University of Hong Kong, Shatin, New Territories, Hong Kong

\section{Shun-Wai Tsang}

Physics Department, The Chinese University of Hong Kong, Shatin, New Territories, Hong Kong

\begin{abstract}
Merton-type models of pricing corporate bonds based on relatively simple default processes cannot generate credit spreads which replicate empirically observed spreads. This article presents an analytical valuation model of corporate discount bond prices to address this problem. The main feature of the model is a dynamic default barrier. Different default scenarios can be incorporated into the valuation model through adjusting the default barrier's dynamics. We derive a closed-form solution of the corporate bond price based on the model as a function of the firm value and short-term interest rate, with time-dependent model parameters. The numerical results calculated from the solution show that the model is capable of producing term structures of credit spreads that are consistent with some empirical findings. This model could provide new insight for future research on corporate bond analysis and credit risk modeling.
\end{abstract}

\section{Introduction}

Black and Scholes (1973) and Merton (1974) have been the pioneers in the pricing of corporate bonds using a contingent-claim framework. In Merton's framework, default occurs only at bond maturity when the firm's asset value is less than its liabilities due to the bond and the firm is insolvent. Default risk is therefore equivalent to a European put option on the firm's asset value. To cope with the possibility of early default before bond maturity, Black and Cox (1976) assume a default-triggering level for the firm's assets whereby default can occur at any time. This trigger level is introduced by considering a safety covenant that protects bondholders. Longstaff and Schwartz (1995) (hereafter LS) extend the corporate bond pricing model of Black and Cox to allow the interest rate to follow the Ornstein-Uhlenbeck process. ${ }^{1}$ The LS model considers a constant

The presentation was improved considerably by the comments of the referee and the Editor. This work is partially supported by the Direct Grant for Research from the Research Grants Council of the Hong Kong Government. The conclusions herein do not represent the views of the Hong Kong Monetary Authority. 
default barrier. Default occurs when the firm's asset value is below the default barrier. Upon default triggered by touching the default barrier, bondholders receive an exogenously given number of risk-free bonds.

However, the LS model suffers from two defects. The first defect is that the payment to bondholders upon early default before bond maturity cannot be prevented from being greater than the firm value. It is because in the LS model the payoff upon default before maturity is exogenously specified and is therefore independent of the level of the default barrier and of the value of the assets. The second defect is that the LS model's pricing solution does not ensure that the firm's asset value upon default is greater than the payment to bondholders at maturity if the default barrier is lower than the face value of the bond. Indeed, the firm can find itself in a solvent position at maturity (ie, its asset value is above the default barrier) but, nevertheless, with assets insufficient to match the face value of the bond. In order to avoid this problem, the default barrier should be restricted at or above the face value of the bond.

Briys and de Varenne (1997) (hereafter BV) develop a pricing model to correct the two defects in the LS model. They define the default barrier as a fixed quantity discounted at the risk-free interest rate up to the maturity date of the corporate bond. ${ }^{2}$ When the firm's asset value drops below this barrier, bondholders receive an exogenously specified fraction of the remaining assets. As a result, the model is characterized by a default barrier with the dynamics of the risk-free interest rate and avoids the limitation of having a constant default barrier as the LS model. Moreover, this default barrier prevents bondholders from receiving a payment greater than the firm value upon default.

LS extend Black and Cox's model to incorporate interest rate risk explicitly into the analysis and also open a new research agenda in the sense that the evolution of the firm's capital structure is no longer tied up with the payoffs of any individual claim on the firm's assets. This feature allows the default barrier to be independent of the state-contingent payoffs of the claim under consideration. The constant default barrier in the LS model corresponds to the total amount of debts issued by the firm, that is kept constant over time. The LS model therefore predicts that the expected leverage ratio will decline exponentially over time. The decline in the expected leverage ratio is however not supported by empirical observations. On the other hand, the BV model defines the default barrier that goes upward with the dynamics of the risk-free interest rate as the bond approaches maturity. As a result, the default barrier is assumed to grow together with the firm value over time and the expected level of leverage is therefore kept constant. This assumption may be appropriate in case the firm is neither willing nor able to reduce its expected level of leverage over time.

Both the LS model and BV model specify the default barriers to follow particular movements, ie, a constant value in the LS model and a fixed quantity discounted at the risk-free interest rate in the BV model. As the movements of the default barriers in these two models are restricted to such simple dynamics, the default barriers are not flexible to incorporate other default scenarios. For exam- 
ple, default could be triggered even though the firm's asset value is above the total amount of debts issued by the firm because of the liquidity problem (such as repayments of short-term debts) faced by the firm. In this case, the default probability is considered to be high and the values of the default barrier could be higher than those defined in the LS and BV models. Similar to the Merton model, both the LS and BV models cannot generate credit spreads which replicate empirically observed spreads.

The main objective of this article is to develop a corporate bond valuation model in which the default barrier is dynamic. The credit spread analysis based on the model show that the use of dynamic default barriers is capable of producing term structures of credit spreads that are consistent with some empirical findings. The dynamics of the default barrier in the model is governed by the stochastic risk-free interest rate and instantaneous variance of the corporate bond value. The contribution of the variance of the corporate bond value to the barrier's dynamics is adjusted by a free parameter $\beta$. When $\beta$ is set equal to zero, the barrier's dynamics depends only on the risk-free interest rate and the proposed model converges to the BV model. When $\beta$ is set to be positive, the default barrier level in the proposed model is higher than that in the BV model. This implies that the default probability of a corporate bond based on the dynamic default barrier with a positive $\beta$ is higher than that in the BV model. The dynamics of the default barrier can thus be adjusted by the parameter $\beta$ to incorporate different default scenarios and to calibrate the model credit spreads with market credit spreads of corporate bonds. The parameter $\beta$ together with the effect of the variance of the corporate bond value can be interpreted as a measure of how much the dynamics of the default barrier deviates from the default barrier assumed in the BV model. ${ }^{3}$ We will also show in the following section that using an appropriate value of $\beta$, the constant default barrier in the LS model can be approximated by the dynamic default barrier. The model in this article is therefore characterised by a dynamic default barrier which extends a constant default barrier in the LS model and a default barrier governed only by the risk-free interest rate in the $\mathrm{BV}$ model.

The dynamics of the short-term interest rate in the proposed model is assumed to follow the Ornstein-Uhlenbeck process, ie, the Vasicek model (Vasicek, 1977). When the firm value touches the default barrier, bondholders receive an exogenously specified number of risk-free discount bonds. Given a default barrier with a positive $\beta$, whose value is larger than a risk-free discount function, the payment upon default is a fraction of the current firm value rather than a fixed amount. This ensures that the firm has sufficient asset value to pay the payment upon default. A non-enforcement of the strict priority rule upon default can be applied to the payoffs to the bondholders. We derive a closedform solution of the bond price as a function of the firm value and interest rate explicitly. The model parameters such as, volatility, correlation, drift and meanlevel of the interest rate are time dependent in the derivation.

The scheme of this article is as follows. In the following section we develop 
the pricing model of discount corporate bonds with a dynamic default barrier, and derive the pricing formula. Numerical results of the term structures of credit spreads based on the pricing formula are shown in Section 3. In the last section we shall summarise our investigation.

\section{Valuation model of corporate bonds}

In the valuation of corporate bonds, we assume a continuous-time framework, and let the short-term interest rate and firm value be stochastic variables. The dynamics of the short-term interest rate $r$ is drawn from the term structure model of Vasicek (1977), ie, the Ornstein-Uhlenbeck process: ${ }^{4}$

$$
\mathrm{d} r=\kappa(t)[\theta(t)-r] \mathrm{d} t+\sigma_{r}(t) \mathrm{d} z_{r}
$$

where the short-term interest rate is mean-reverting to a long-run mean $\theta(t)$ at speed $\kappa(t)$, and $\sigma_{r}(t)$ is the volatility of $r$. The firm value $A$ is assumed to follow a lognormal diffusion process:

$$
\mathrm{d} A=\mu(t) A \mathrm{~d} t+\sigma_{A}(t) A \mathrm{~d} z_{A}
$$

where $\mu(t)$ and $\sigma_{A}(t)$ are the drift and volatility of the firm value respectively. The Wiener processes $\mathrm{d} z_{A}$ and $\mathrm{d} z_{r}$ are correlated with

$$
\mathrm{d} z_{A} \mathrm{~d} z_{r}=\rho(t) \mathrm{d} t
$$

and the correlation coefficient $\rho$ is also assumed to be time-dependent. In the absence of taxes and bankruptcy costs, the firm value is assumed to be independent of the capital structure of the firm. This is the standard assumption that the Modigliani-Miller (1958) theorem holds.

We let the price of a discount corporate bond be $P(A, r, t)$ where $t$ is the remaining time to maturity. Using Ito's lemma and the standard no-arbitrage arguments, the partial differential equation governing the bond is

$$
\begin{aligned}
& \frac{\partial P}{\partial t}=\frac{1}{2} \sigma_{A}^{2}(t) A^{2} \frac{\partial^{2} P}{\partial A^{2}}+\frac{1}{2} \sigma_{r}^{2}(t) \frac{\partial^{2} P}{\partial r^{2}}+\rho_{A r}(t) \sigma_{A}(t) \sigma_{t}(t) A \frac{\partial^{2} P}{\partial A \partial r}+r A \frac{\partial P}{\partial A} \\
& +[\kappa(t) \theta(t)-\kappa(t) r-\lambda] \frac{\partial P}{\partial r}-r P
\end{aligned}
$$

where $\lambda$ is the market price of interest rate risk. ${ }^{5}$ In the derivation of the pricing solution of equation (4), $\lambda$ is absorbed into the term $\kappa(t) \theta(t)$. The value of the corporate bond is obtained by solving equation (4) subject to the final payoff condition and the boundary condition imposed by the default barrier.

Given a corporation, the default barrier represents either a safety covenant which is a contractual agreement to let bondholders have the right to bankrupt (or force reorganisation of) the firm when its asset value falls below a certain level, or a default point determined mainly by the firm's short-term liabilities. As a corporate bond has some time to maturity, its default barrier level is likely to 
change with time. The dynamics of a default barrier governed by risk-free interest rates have been discussed by Black and Cox (1976) and BV in regard to constant and stochastic interest rates respectively. In order to incorporate a dynamic default barrier into the corporate bond valuation model, we propose the default barrier $H(r, t)$ to have dynamics which is determined by the short-term interest rate and the instantaneous variance of the corporate bond value. The default barrier is specified in the following way:

$$
H(r, t)=X Q(r, t) \exp \left[\beta c_{1}(t)\right]
$$

where $X$ is the pre-defined value of the default barrier, $Q(r, t)$ is the risk-free discount function according to the Vasicek model, $c_{1}(t)$, is defined as:

$$
\begin{aligned}
& c_{1}(t)=\int_{0}^{t} \mathrm{~d} \tau\left[\frac{1}{2} \sigma_{A}^{2}(\tau)+\rho(\tau) \sigma_{A}(\tau) \sigma_{r}(\tau) c_{4}(\tau)+\frac{1}{2} \sigma_{r}^{2}(\tau) c_{4}^{2}(\tau)\right] \\
& c_{4}(t)=\exp \left[-\int_{0}^{t} \mathrm{~d} \tau \kappa(\tau)\right] \int_{0}^{t} \mathrm{~d} \tau \exp \left[\int_{0}^{\tau} \mathrm{d} \tau^{\prime} \kappa\left(\tau^{\prime}\right)\right]
\end{aligned}
$$

and $\beta$ is a real number parameter to adjust the contribution of $c_{1}(t)$. The function $c_{1}(t)$ is the integrated instantaneous variance of the corporate bond function over the life of the corporate bond, and the function $c_{4}^{2}(t) \sigma_{r}^{2}(t)$ is the instantaneous variance of the Vasicek model risk-free zero-coupon bond with time to maturity $t$. The movement of the barrier can therefore be interpreted as a mean drift (adjusted by $\beta$ ) arising from the dynamics of $r$ and $P(A, r, t)$. The firm value volatility $\sigma_{A}(t)$ is incorporated into the barrier dynamics through $c_{1}(t)$. It is also for analytically convenience to choose the function $c_{1}(t)$ in order to obtain the closed-form solution in equation (9) below. It is noted that the parameter $\beta$ and the function $c_{1}(t)$ can be viewed as a combined parameter that adjusts the dynamics of the default barrier. The default barrier levels with $\beta=0,1.15$ and 1.5 , and parameters $X=1, \sigma_{A}=0.25, \sigma_{r}=0.0316, \rho=-0.25, r=5 \%, \theta=5 \%$ and $\kappa=1$ are illustrated in Figure 1. The "natural" time is from left to right. If $X$ is set equal to the face value $F$ of the bond, the default barrier converges to $F$ at bond maturity (ie, $t=0$ ).

When the parameter $\beta$ is set to be zero, the barrier follows the dynamics of a risk-free bond, ie, recovering the BV model. Figure 1 demonstrates that the default barrier level in the BV model (ie, $\beta=0$ ) decreases with time to maturity according to the risk-free bond function $Q(r, t)$. Given a positive $\beta$, the function $c_{1}(\mathrm{t})$ which is positive offsets the decreasing effect of the risk-free bond function. ${ }^{6}$ Figure 1 shows that the values of the dynamic default barrier defined in equation (5) increase with positive $\beta$, that implies higher default probability of a corporate bond. When $\beta$ is set equal to 1.15 , the default barrier is almost flat over time. It is similar to a constant barrier $X$ specified in the LS model. Firms which are subject to different default scenarios may be represented by the dynamic default barrier based on different $\beta$. A firm with $\beta=1.5$ has a dynamic 
FIGURE I Default barrier level with different $\beta$.

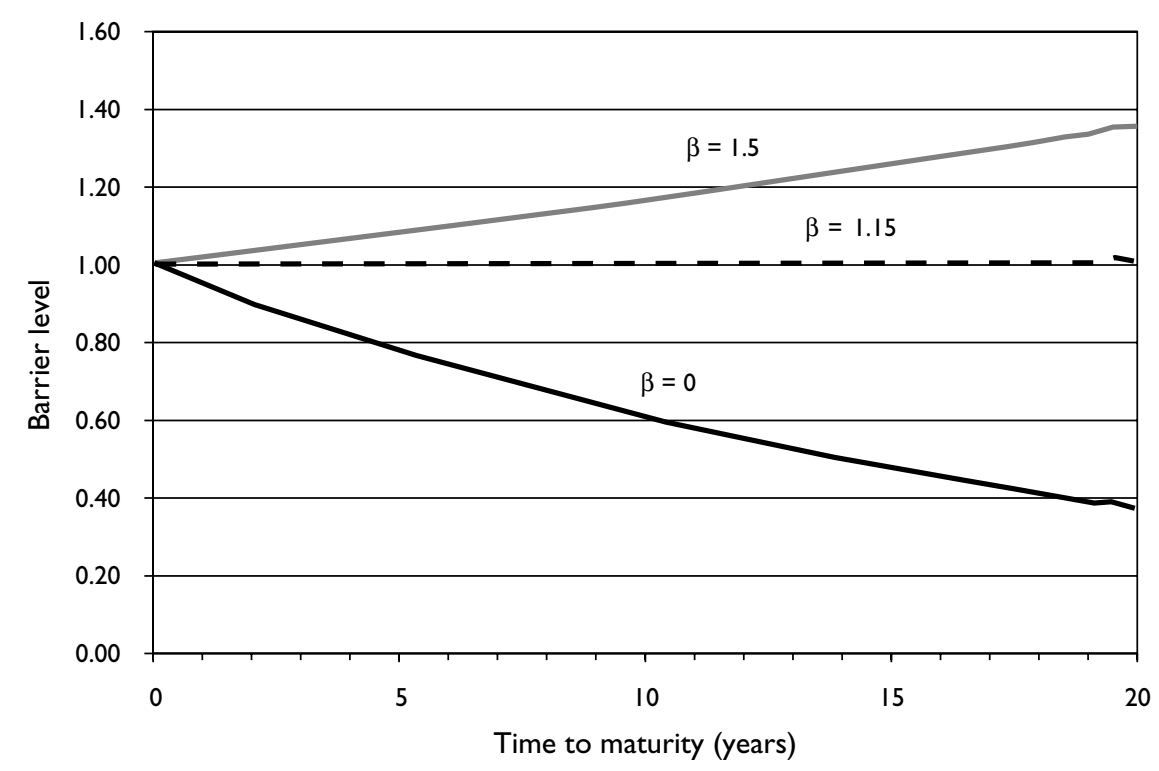

The default barriers are plotted as a function of time to maturity $t$ with $\beta=0,1.15$ and I.5. The "natural" time movement is from right to left. The other parameters used are $X=1, \sigma_{A}=0.25, \sigma_{r}=0.0316, \rho=$ $-0.25, r=5 \%, \theta=5 \%$ and $\kappa=1$.

default barrier that is higher than the face value $F$ (which is set equal to $X$ ) of the bond at longer maturities (see Figure 1). The firm can be considered to have significant amounts of short-term liabilities that let the firm easily face a short-term liquidity problem. Default could thus be triggered by the liquidity problem. Regarding $\beta=1.15$, the approximately flat default barrier presents a firm with constant total outstanding debts over time. Default occurs when the firm value falls below the value of total debts. It is the default scenario defined in the LS model. In the BV model (ie, $\beta=0$ ), a firm's default is its long-term debts. The default barrier is discounted at the rate of the short-term interest rate with time to maturity. The default risk is thus lower than that based on $\beta=1.15$ and 1.5. Numerical results of credit spreads obtained from the dynamic default barriers illustrated in Figure 1 are presented in the next section.

When the firm value breaches the barrier $H(r, t)$, bankruptcy occurs before bond maturity at $t=0$. The payoffs to bondholders are specified by:

$$
P(A=H, r, t)=\alpha_{1} X Q(r, t) \quad t>0 ; 0 \leq \alpha_{1} \leq 1
$$

For $\beta \geq 0$, the payoffs to bondholders upon default should always be less than the firm value ${ }^{7}$ as $c_{1}(t)$ is positive. On the other hand, if the firm value has never breached the default barrier, the payoffs to bondholders at the bond maturity are: 


$$
\begin{array}{ll}
P(A, r, t=0)=F & A \geq F \\
P(A, r, t=0)=\alpha_{2} A & H<A<F ; 0 \leq \alpha_{2} \leq 1
\end{array}
$$

The parameters $\alpha_{1}$ and $\alpha_{2}$ determine the payoffs upon default. If $\alpha_{1}=\alpha_{2}=1$, the strict priority rule is enforced and the shareholders of the firm receive nothing. On the other hand, if $\alpha_{1}$ and $\alpha_{2}$ are between zero and one, it implies the nonenforcement of the strict priority rule. In a case of $X=F$, as the default barrier converges to $F$ at bond maturity, the condition in equation (7) will be sufficient to determine the payoff at default at all times and the condition in equation (8) is not necessary. If $\alpha_{1}$ is equal to 1 in this case, the payoff at default will be a riskfree discount bond with face value $F$ and the corporate bond will become risk free.

Other than the dynamics of the default barrier, the valuation framework here preserves the main features of the BV model. The features are as follows:

$\square$ a bundle of risk-free bonds will be received by the bondholders upon default before bond maturity;

$\square$ as long as $0 \leq \alpha_{1} \leq 1$ and $\beta \geq 0$, the payment to bondholders is no greater than the firm value upon default;

$\square$ the model is reduced to the Merton model when $X$ is set to be zero; and

$\square$ the deviation from the absolute priority rule is allowed.

The solution of equation (4) subject to equation (7) and (8) is

$$
\begin{aligned}
P=F Q & \left\{\alpha_{2} \frac{l}{Q}\left[N\left(d_{1}\right)-N\left(d_{2}\right)\right]-\alpha_{1} \frac{l}{q}\left[N\left(d_{1}+\sqrt{2 c_{1}}\right)-N\left(d_{2}+\sqrt{2 c_{1}}\right)\right]\right. \\
& -\alpha_{2} l q^{-(\beta+1)} Q^{\beta} \mathrm{e}^{\beta(\beta+1) c_{1}}\left[N\left(d_{3}\right)-N\left(d_{4}\right)\right] \\
& +\alpha_{1} l q^{-\beta} Q^{\beta-1} \mathrm{e}^{\beta(\beta-1) c_{1}}\left[N\left(d_{3}+\sqrt{2 c_{1}}\right)-N\left(d_{4}+\sqrt{2 c_{1}}\right)\right] \\
& +\left(1-\frac{\alpha_{1} l}{q}\right) N\left(-d_{1}-\sqrt{2 c_{1}}\right) \\
& \left.-\left(q^{1-\beta}-\alpha_{1} l q^{-\beta}\right) Q^{\beta-1} \mathrm{e}^{\beta(\beta-1) c_{1}} N\left(-d_{3}-\sqrt{2 c_{1}}\right)\right\}+\alpha_{1} X Q
\end{aligned}
$$

where $l=A / F$ is the asset-to-liability ratio, $q=A / X$ is an early default ratio, and

$$
\begin{array}{ll}
d_{1}=\frac{-\ln l+\ln Q-c_{1}}{\sqrt{2 c_{1}}} & d_{2}=\frac{-\ln q+\ln Q-c_{1}}{\sqrt{2 c_{1}}} \\
d_{3}=\frac{-\ln l+2 \ln q-\ln Q-(2 \beta+1) c_{1}}{\sqrt{2 c_{1}}} & d_{4}=\frac{\ln q-\ln Q-(2 \beta+1) c_{1}}{\sqrt{2 c_{1}}}
\end{array}
$$


The detailed derivation of the solution in equation (9) is given in the Appendix. It is easy to show from equation (9) that the corporate bond price is equal to the recovery value $\alpha_{1} X Q(r, t)$ when $A$ breaches the barrier, ie,

$$
A=X Q(r, t) \exp \left[\beta c_{1}(t)\right]
$$

Because of the final condition in equation (8), the corporate bond price gives the same results of the Merton model if the default barrier is set at zero (ie, $X=0$ ) and $\alpha_{2}$ is equal to one. In the case of $X=F$, the default conditions (ie, $A<F$ ) at bond maturity are the same in both the Merton model and the model with dynamic default barriers. While there is no early default in the Merton model, the dynamic default barriers would trigger default before bond maturity and thus cause higher probability of default than that obtained in the Merton model. The credit spreads of corporate bonds based on equation (9) are illustrated in the following section.

\section{Credit spread analysis}

The credit spread $C_{s}$ of a corporate discount bond price $P(A, r, t)$ with time to maturity $t$ and face value $F$ is given as

$$
C_{s}(A, r, t)=-\frac{1}{t} \ln \frac{P(A, r, t)}{F Q(r, t)}
$$

The term structures of credit spreads for a low leveraged firm, with $A=10.5$ and $F=1$, are illustrated in Figure 2 using $\beta=0,1.15$ and 1.5 . The pre-defined value of the default barrier $X$ is assumed to be 1 such that the value of the default barrier converges to the face value $F$ of the bond at bond maturity. This means that the condition in equation (7) is sufficient to determine the payoff at default at all times. Other parameters used in the calculations are $\sigma_{A}=0.25, \sigma_{r}=0.0316, \rho=$ $-0.25, r=5 \%, \theta=5 \%, \kappa=1$ and $\alpha_{1}=0.48$. Bondholders receive $\alpha_{1}=48 \%$ of the risk-free discount bond value with face value $F$ upon default. For $\beta=0$, the term structure of credit spreads with $\alpha_{1}=0.74$ is used to show the effect of the recovery rate. The asset volatility of $\sigma_{A}=0.25$ is close to the median for publicly listed firms. The term structure of credit spreads based on the Merton model (ie, $X \rightarrow 0$ and $\alpha_{2}=1$ ) is also shown in Figure 2 for comparison purposes. The debt ratio $F / A=9.5 \%$ in this illustration is similar to the market-value debt ratio of firms whose senior debt ratings from Standard and Poor's (2001) are AA. The expected recovery rate for senior unsecured debt is typically $48 \%$ of the bond value according to Carty and Lieberman (1998). The use of $\alpha_{1}$ in calculations is close to the empirical observations.

The term structures based on the proposed model exhibit upward slopes at short maturities in Figure 2. At longer maturities, their shapes are either flat or upward-sloping depending on the values of $\beta$. Intuitively, this is because the probability that the firm reaches the default barrier increases over time. The shapes of the two term structures with $\beta=1.15$ and 1.5 exhibit upward slopes at 
FIGURE 2 Credit spread term structures of low leveraged (AA-rated) firm.

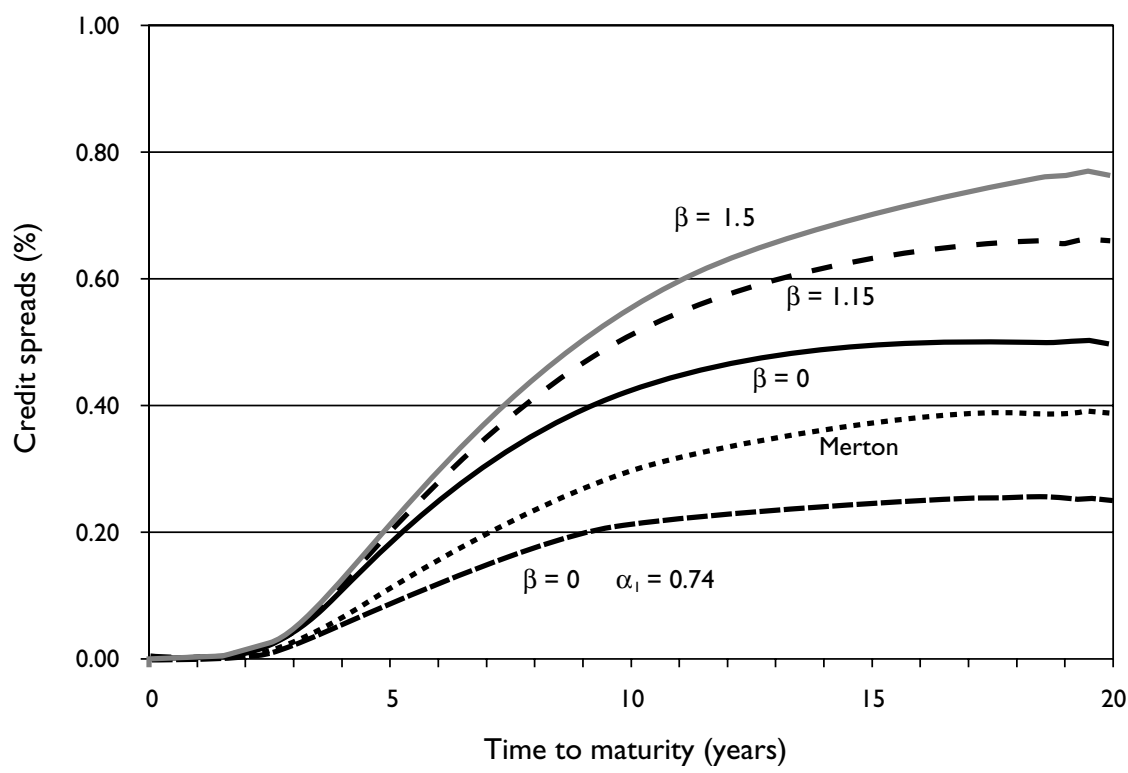

The credit spreads of a low leverage (AA-rated) firm with $A=10.5$ and $X=F=I$ are plotted using $\beta=$ $0, \mathrm{I} .15$ and 1.5. The other parameters used are $\sigma_{A}=0.25, \sigma_{r}=0.0316, \rho=-0.25, r=5 \%, \theta=5 \%, \kappa=1$ and $\alpha_{1}=0.48$ (also $\alpha_{1}=0.74$ for $\beta=0$ ). The term structure of a corporate bond based on the Merton model with $\alpha_{2}=\mathrm{I}$ and the same other parameters is illustrated for comparison.

longer maturities. The term structure obtained by using $\beta=1.15$ (that corresponds to an almost constant default barrier) can be approximated as the result based on the LS model. Both the term structures are similar to their empirical studies in Sarig and Warga (1989) and Fons (1994). Sarig and Warga (1989) find that the term structure of credit spreads is upward sloping for low leveraged firms using pure discount bonds. Fons (1994) find that bonds rated Aa (Moody's rating) exhibit a significant positive relation between spreads and maturity based on the market data of 374 Aa-rated bonds with maturities longer than 20 years. The shape of the term structure with $\beta=0$ (ie, the BV model) exhibits flat at longer maturities, that is similar to that obtained from the Merton model.

Figure 2 also shows that the credit spreads based on dynamic default barriers increase with positive $\beta$ and are higher than those obtained from the Merton model that does not capture early default risk. This demonstrates that the levels of the default barrier with larger $\beta$ imply higher early default risk as discussed in the previous section. However, at longer maturities, the differences between the credit spreads for $\beta=0$ and $\beta=1.5$ are about $0.25 \%$, which are significant compared with the average credit spread of $0.7 \%$ of a bond with $\beta=1.5$. When a higher recovery rate (ie, $\alpha_{1}=0.74$ ) is used, the credit spreads are reduced by more or less one half of those based on $\alpha_{1}=0.48$. They are even lower than those based on the Merton model. This shows that both the default process and 
FIGURE 3 Credit spread term structures of medium leveraged (BBB-rated) firm.

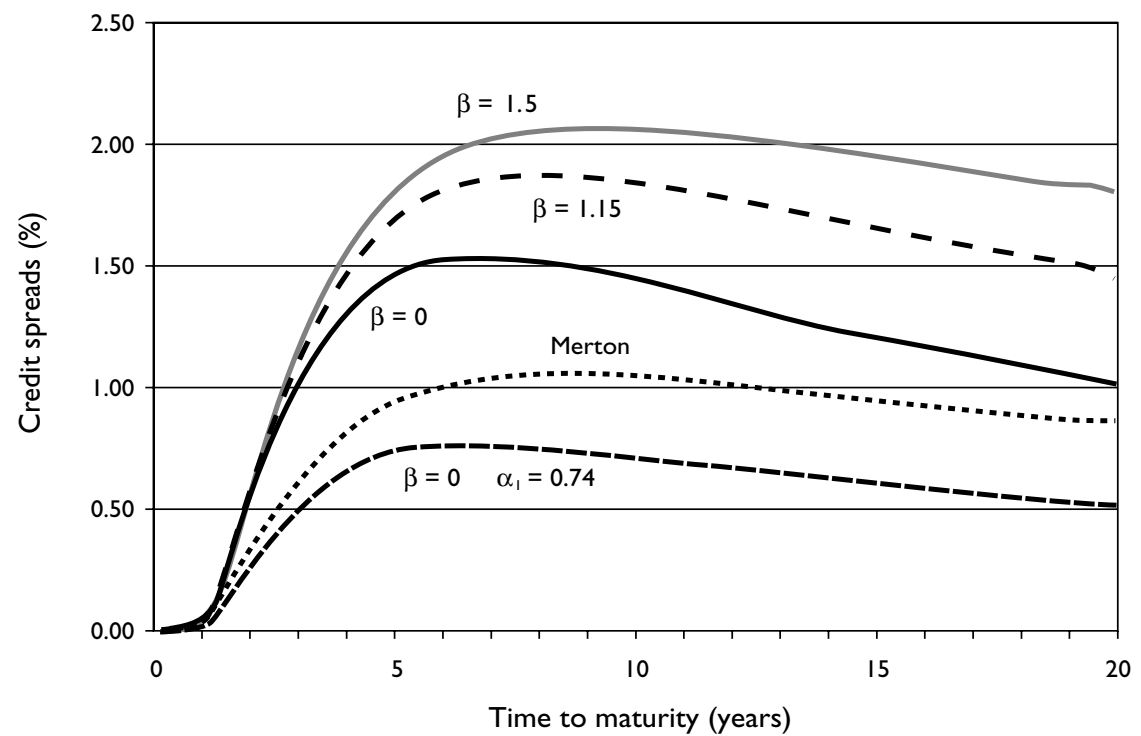

The credit spreads of a medium leveraged (BBB-rated) firm with $A=3.17$ and $X=F=1$ are plotted using $\beta=0$, I.I5 and I.5. The other parameters used are $\sigma_{A}=0.25, \sigma_{r}=0.0316, \rho=-0.25, r=5 \%, \theta=$ $5 \%, \kappa=I$ and $\alpha_{1}=0.48$ (also $\alpha_{1}=0.74$ for $\beta=0$ ). The term structure of a corporate bond based on the Merton model with $\alpha_{2}=\mathrm{I}$ and the same other parameters is illustrated for comparison.

FIGURE 4 Credit spread term structures of highly leveraged (BB-rated) firm.

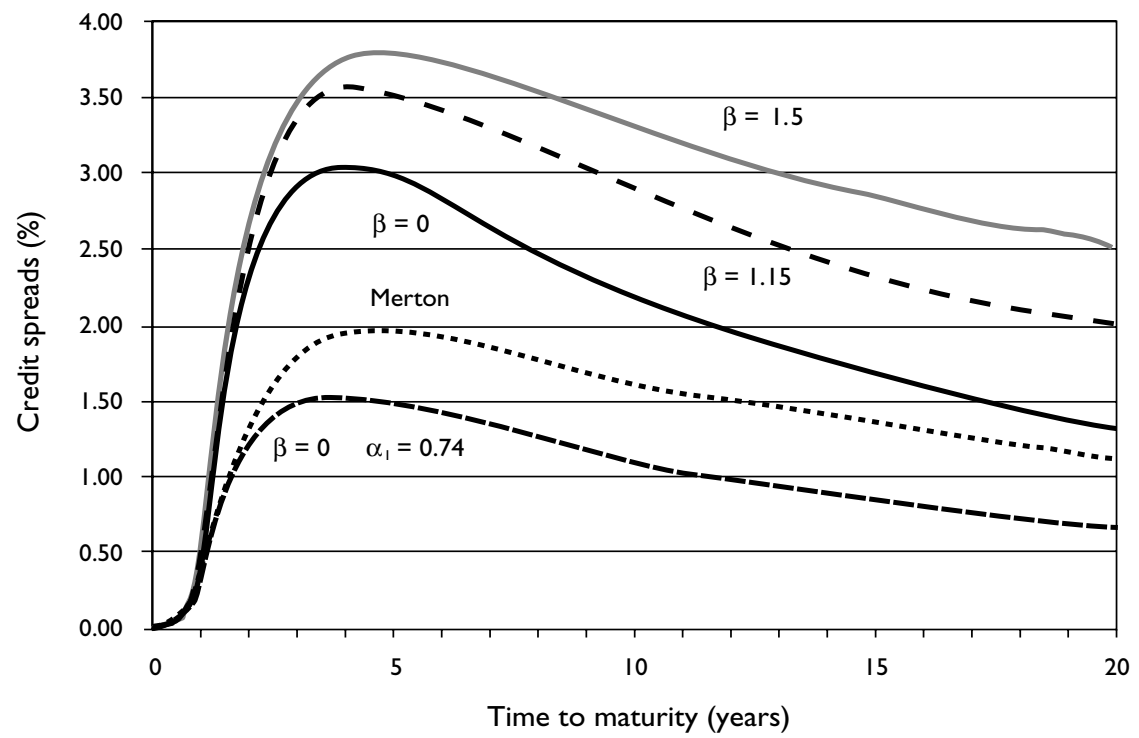

Credit spreads of a highly leveraged (BB-rated) firm with $A=2.0$ and $X=F=I$ are plotted using $\beta=0$, I.I5 and I.5. The other parameters used are $\sigma_{A}=0.25, \sigma_{r}=0.0316, \rho=-0.25, r=5 \%, \theta=5 \%, \kappa=I$ and $\alpha_{1}=0.48$ (also $\alpha_{1}=0.74$ for $\beta=0$ ). The term structure of a corporate bond based on the Merton model with $\alpha_{2}=\mathrm{I}$ and the same other parameters is illustrated for comparison. 
the recovery rate have impact on the credit spreads. Given $X=F$, if there is no loss given default (ie, $\alpha_{1}=1$ ), the corresponding corporate bond will become risk free and its credit spreads will be zero.

The term structures of credit spreads for a medium leveraged firm, with $A=$ 3.17 and $F=X=1$, are illustrated in Figure 3 using $\beta=0,1.15$ and 1.5 and the Merton model. The debt ratio $F / A=31.5 \%$ is similar to the market-value debt ratios of firms whose senior debt ratings from Standard and Poor's are BBB. Other parameters are the same as those used in Figure 2. The depicted term structures exhibit upward slopes at short maturities. At longer maturities, their shapes display different degrees of downward-sloping depending on the values of $\beta$. The shapes are consistent with the empirical finding by Sarig and Warga (1989) that the term structure is humped for medium leveraged firms. The shape of the term structure with $\beta=1.5$ exhibits a gentle downward slope at longer maturities. It does not however match with the empirical finding by Fons (1994) that the term structure of Baa-rated bonds is gently upward-sloping.

Similar to Figure 2, Figure 3 also shows that the credit spreads based on dynamic default barriers increase with positive $\beta$ and are higher than those obtained from the Merton model. The differences between the credit spreads for $\beta=0$ and $\beta=1.5$ are $0.75 \%$ on average and are more at longer maturities. Among the credit spreads presented in Figure 3, the credit spreads based on $\beta=$ 1.5 agree better with Altman's findings (Altman 1989) on BBB-rated bonds and Fons' findings on Baa-rated bonds, which are reported to have credit spreads averaging $1.77 \%$ and $1.85 \%$ respectively. The credit spreads based on $\beta=0$ and $\alpha_{1}=0.74$ also show that the recovery rate has impact on the credit spreads.

Figure 4 shows the term structures of credit spreads for a highly leveraged firm, with $A=2.0$ and $F=X=1$, using $\beta=0,1.15$ and 1.5 and the Merton model. The debt ratio $F / A=50 \%$ is similar to the market-value debt ratios of firms whose senior debt ratings from Standard and Poor's are BB. Other parameters are the same as those used in Figure 2. The depicted term structures exhibit steep upward slopes at short maturities and are downward-sloping for maturities longer than a few years. The shapes are consistent with the empirical findings of the downward-sloping term structures of highly leveraged firms and speculative grade (Ba-rated) bonds reported by Sarig and Warga (1989) and Fons (1994) respectively. The phenomenon of high credit spreads at short maturities is called "crisis-at-maturity" by Johnson (1967). ${ }^{8}$ This downward slope is generated by high initial default probabilities which are expected to decrease over time as the firm survives.

Similar to Figures 2 and 3, Figure 4 also shows that the credit spreads based on dynamic default barriers increase with positive $\beta$. The differences between the credit spreads for $\beta=0$ and $\beta=1.5$ are about $1 \%$. Among the credit spreads presented in Figure 4, the credit spreads based on $\beta=1.5$ again agree better with Fons' findings on Ba-rated bonds, which are reported to have credit spreads of averaged $3.37 \%$. Other term structures present values of credit spreads lower than the empirical credit spreads. 
FIGURE 5 Credit spread term structures of low leveraged firm with high asset volatility.

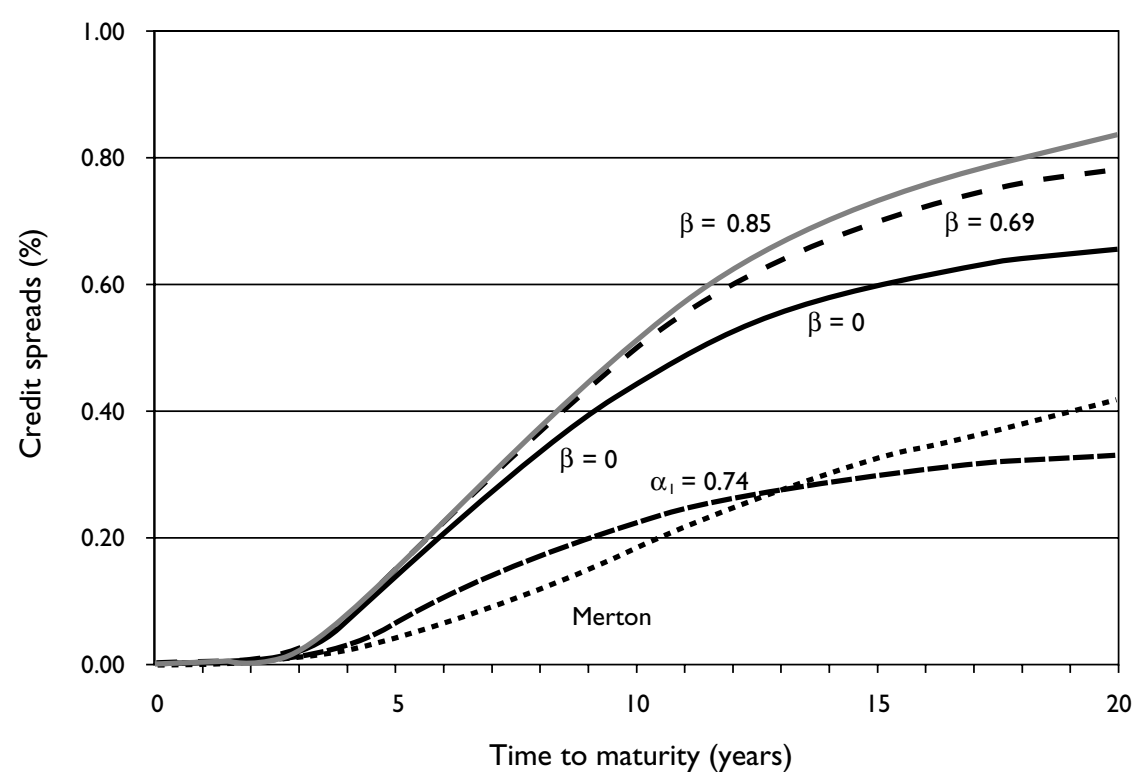

Credit spreads of a low leveraged firm with $A=10.5, \sigma_{A}=0.4$ and $X=F=I$ are plotted using $\beta=0,0.69$ and 0.85 . The other parameters used are $\sigma_{r}=0.0316, \rho=-0.25, r=5 \%, \theta=5 \%, \kappa=I$ and $\alpha_{1}=0.48$ (also $\alpha_{1}=0.74$ for $\beta=0$ ). The term structure of a corporate bond based on the Merton model with $\alpha_{2}=1$ and the same other parameters is illustrated for comparison.

The term structures of credit spreads for a low leveraged firm, with $A=10.5$, $F=X=1$ and high asset volatility $\sigma_{A}=0.4$, are illustrated in Figure 5 using $\beta=$ $0,0.69$ and 0.85 and the Merton model. This firm is similar to a small/mediumsized technology company. Because a different $\sigma_{A}$ is used, the values of $\beta$ are different from those used in previous figures such that the structures of the default barriers are close to those in Figure 1. Other parameters are the same as those used in Figure 2. The depicted term structures in Figure 5 have slightly higher values than those shown in Figure 2, which are based on a low leveraged firm with medium asset volatility. This shows that credit spreads of a low leveraged firm are not sensitive to high asset volatility. As the asset value of such a firm is much higher than the default barriers and the face value of the bond, high asset volatility will not increase its default risk materially.

The term structures of credit spreads for a highly leveraged firm, with $A=2$, $F=X=1$ and high asset volatility $\sigma_{A}=0.4$, are illustrated in Figure 6 using $\beta=$ $0,0.69$ and 0.85 and the Merton model. This firm is similar to a large-sized technology company. Other parameters are the same as those used in Figure 2. The values of the credit spreads at short maturities based on the dynamic default barriers in Figure 6 are almost double of those in the corresponding term structures shown in Figure 4, which are based on a highly leveraged firm with medium 
FIGURE 6 Credit spread term structures of highly leveraged firm with high asset volatility.

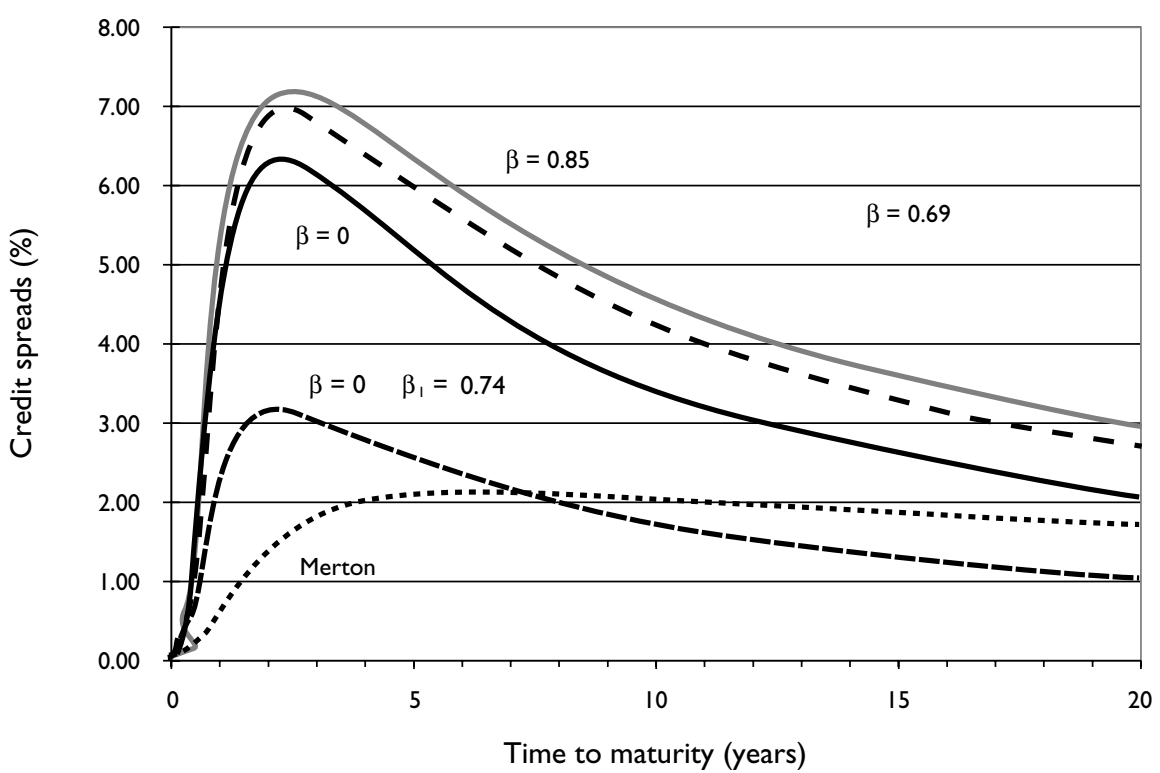

Credit spreads of a highly leveraged firm with $A=2, \sigma_{A}=0.4$ and $X=F=1$ are plotted using $\beta=0,0.69$ and 0.85 . The other parameters used are $\sigma_{r}=0.0316, \rho=-0.25, r=5 \%, \theta=5 \%, \kappa=I$ and $\alpha_{1}=0.48$ (also $\alpha_{1}=0.74$ for $\beta=0$ ). The term structure of a corporate bond based on the Merton model with $\alpha_{2}=1$ and the same other parameters is illustrated for comparison.

asset volatility. The results show that credit spreads of a highly leveraged firm are sensitive to high asset volatility. As the asset value of such a firm is close to default barriers, high asset volatility will increase its default risk significantly, in particular at short maturities. Conversely, the credit spreads based on the Merton model, in which default is determined at bond maturity, are much less sensitive to high asset volatility.

Additional calculations are conducted for both low and highly leveraged firms with low asset volatility of $\sigma_{A}=0.08$. The values of credit spreads obtained from both the Merton model and the model with dynamic default barriers are however close to zero. This is a common deficiency in the structural models that they cannot capture default risk of firms with low asset volatility, for example banks and food retailers.

In summary, the numerical results show that incorporating a dynamic default barrier gives the basic shapes of the term structures of credit spreads similar to some empirical findings. The credit spreads increase with positive $\beta$ that represents different default scenarios in different firms. The term structures obtained by the dynamic default barriers with appropriate $\beta$ (such as $\beta=1.5$ used in the above analysis) for different leveraged firms could broadly match with the empirical findings for similarly rated corporate bonds (ie, AA, BBB and BB). 
However, the prediction by the proposed model that credit spreads are close to zero at very short maturities contradicts the findings of Fama (1986) from commercial paper credit spreads. The problem of downward-biased credit spreads at short maturities is common to all contingent-claims pricing models which assume continuous dynamics. Therefore, criticism of the models based on low credit spreads at short maturities is that of the underlying assumptions. Zhou (1997) and Madan and Unal (2000) derive models in which the short-term credit spreads are non-trivial.

\section{Conclusion}

This paper develops a corporate bond valuation model that incorporates a dynamic default barrier which is governed by stochastic interest rates and the variance of the corporate bond value. Different default scenarios can be incorporated into the valuation model through adjusting the default barrier's dynamics. We derive a closed-form solution of the corporate bond price as a function of firm value and the short-term interest rate, with time-dependent model parameters. The numerical results show that the model is capable of producing term structures of credit spreads which are broadly consistent with some empirical findings. The model could therefore provide new insight for future research on corporate bonds analysis and credit risk modeling. More detailed empirical comparisons between the actual credit spreads and the model credit spreads are left to future research.

\section{Appendix}

The price of a corporate bond, which is a function of the firm value $A$, the shortterm interest rate $r$ and the time to maturity $t$ is governed by the partial differential equation

$$
\begin{aligned}
\frac{\partial P(A, r, t)}{\partial t} & =\frac{1}{2} \sigma_{A}^{2}(t) A^{2} \frac{\partial^{2} P(A, r, t)}{\partial A^{2}}+\rho(t) \sigma_{r}(t) \sigma_{A}(t) A \frac{\partial^{2} P(A, r, t)}{\partial r \partial A} \\
& +\frac{1}{2} \sigma_{r}^{2}(t) \frac{\partial^{2} P(A, r, t)}{\partial r^{2}}+r A \frac{\partial P(A, r, t)}{\partial A} \\
& +\kappa(t)[\theta(t)-r] \frac{\partial P(A, r, t)}{\partial r}-r P(A, r, t)
\end{aligned}
$$

To solve this partial differential equation, we first rewrite it in terms of the variable $x=\ln A$ as follows: 


$$
\begin{aligned}
\frac{\partial P(x, r, t)}{\partial t} & =\frac{1}{2} \sigma_{A}^{2}(t) \frac{\partial^{2} P(x, r, t)}{\partial x^{2}}+\rho(t) \sigma_{r}(t) \sigma_{A}(t) \frac{\partial^{2} P(x, r, t)}{\partial r \partial x} \\
& +\frac{1}{2} \sigma_{r}^{2}(t) \frac{\partial^{2} P(x, r, t)}{\partial r^{2}}+\left[r-\frac{1}{2} \sigma_{A}^{2}(t)\right] \frac{\partial P(x, r, t)}{\partial x} \\
& +\kappa(t)[\theta(t)-r] \frac{\partial P(x, r, t)}{\partial r}-r P(x, r, t) \\
& \equiv H(t) P(x, r, t)
\end{aligned}
$$

We define the evolution operator $U(t, 0) \equiv U_{0}(t, 0) U_{I}(t, 0)$ such that

$$
P(x, r, t)=U(t, 0) P(x, r, 0)=U_{0}(t, 0) U_{I}(t, 0) P(x, r, 0)
$$

Inserting equation (A3) into equation (4) yields the evolution equations

$$
\begin{array}{ll}
H_{0}(t) U_{0}(t, 0)=\frac{\partial}{\partial t} U_{0}(t, 0), & U_{0}(0,0)=1 \\
H_{I}(t) U_{I}(t, 0)=\frac{\partial}{\partial t} U_{I}(t, 0), & U_{I}(0,0)=1
\end{array}
$$

where

$$
\begin{aligned}
H_{0}(t) & =\frac{1}{2} \sigma_{A}^{2}(t) \frac{\partial^{2}}{\partial x^{2}}+\rho(t) \sigma_{r}(t) \sigma_{A}(t) \frac{\partial^{2}}{\partial r \partial x}+\frac{1}{2} \sigma_{r}^{2}(t) \frac{\partial^{2}}{\partial r^{2}} \\
& +r \frac{\partial}{\partial x}-\kappa(t) r \frac{\partial}{\partial r}
\end{aligned}
$$

and $H_{1} \equiv U_{0}(t, 0)^{-1}\left[H(t)-H_{0}(t)\right] U_{0}(t, 0)$. It is not difficult to show that the operator $H_{0}(t)$ can be rewritten in the following form:

$$
H_{0}(t)=\sum_{i=1}^{5} a_{i}(t) \hat{J}_{i}
$$

where

$$
\begin{aligned}
& \hat{J}_{1}=\frac{\partial^{2}}{\partial x^{2}} \quad \hat{J}_{2}=\frac{\partial^{2}}{\partial r^{2}} \quad \hat{J}_{3}=\frac{\partial^{2}}{\partial r \partial x} \quad \hat{J}_{4}=r \frac{\partial}{\partial x} \quad \hat{J}_{5}=r \frac{\partial}{\partial r} \\
& \alpha_{1}(t)=\frac{1}{2} \sigma_{A}^{2}(t) \quad \alpha_{2}(t)=\frac{1}{2} \sigma_{r}^{2}(t) \quad \alpha_{3}(t)=\rho(t) \sigma_{r}(t) \sigma_{A}(t) \\
& \alpha_{4}(t)=1 \quad \alpha_{5}(t)=-\kappa(t)
\end{aligned}
$$

The operators $\hat{J}_{i}$ form a solvable Lie algebra: 


$$
\begin{aligned}
& {\left[\hat{J}_{2}, \hat{J}_{3}\right]=\left[\hat{J}_{1}, \hat{J}_{k}\right]=0 \quad(k=2,3,4 \text { and } 5)} \\
& {\left[\hat{J}_{2}, \hat{J}_{4}\right]=2 \hat{J}_{3}, \quad\left[\hat{J}_{2}, \hat{J}_{5}\right]=2 \hat{J}_{2}, \quad\left[\hat{J}_{3}, \hat{J}_{4}\right]=\hat{J}_{1}} \\
& {\left[\hat{J}_{3}, \hat{J}_{5}\right]=\hat{J}_{3}, \quad\left[\hat{J}_{4}, \hat{J}_{5}\right]=-\hat{J}_{4}}
\end{aligned}
$$

According to the Wei-Norman theorem (Wei and Norman, 1963), the evolution operator $U_{0}(t, 0)$ can be expressed in the form

$$
\begin{aligned}
U_{0}(t, 0)= & \exp \left[c_{4}(t) \hat{J}_{4}\right] \exp \left[c_{1}(t) \hat{J}_{1}\right] \exp \left[c_{2}(t) \hat{J}_{2}\right] \times \\
& \exp \left[c_{5}(t) \hat{J}_{5}\right] \exp \left[c_{3}(t) \hat{J}_{3}\right]
\end{aligned}
$$

where the coefficients $c_{i}(t)$ are to be determined. Then by direct differentiation with respect to $t$, we obtain

$$
\frac{\partial}{\partial t} U_{0}(t, 0)=\sum_{i=1}^{5} h_{i}(t) \hat{J}_{i} U_{0}(t, 0)
$$

with

$$
\begin{aligned}
& h_{1}(t)=\frac{\partial c_{1}}{\partial t}+c_{4}^{2} \frac{\partial c_{2}}{\partial t}+2 c_{2} c_{4}^{2} \frac{\partial c_{5}}{\partial t}-c_{4} \exp \left(-c_{5}\right) \frac{\partial c_{3}}{\partial t} \\
& h_{2}(t)=\frac{\partial c_{2}}{\partial t}+2 c_{2} \frac{\partial c_{5}}{\partial t} \\
& h_{3}(t)=\exp \left(-c_{5}\right) \frac{\partial c_{3}}{\partial t}-2 c_{4} \frac{\partial c_{2}}{\partial t}-4 c_{2} c_{4}^{2} \frac{\partial c_{5}}{\partial t} \\
& h_{4}(t)=\frac{\partial c_{4}}{\partial t}-c_{4} \frac{\partial c_{5}}{\partial t} \\
& h_{5}(t)=\frac{\partial c_{5}}{\partial t}
\end{aligned}
$$

Substituting equations (A7), (A10) and (A11) into (A4), and comparing the two sides, we find after simplification

$$
\begin{aligned}
& c_{5}(t)=\int_{0}^{t} \mathrm{~d} t^{\prime} a_{5}\left(t^{\prime}\right) \\
& c_{4}(t)=\exp \left[c_{5}(t)\right] \int_{0}^{t} \mathrm{~d} t^{\prime} a_{4}\left(t^{\prime}\right) \exp \left[-c_{5}\left(t^{\prime}\right)\right]
\end{aligned}
$$




$$
\begin{aligned}
& c_{3}(t)=\int_{0}^{t} \mathrm{~d} t^{\prime}\left[a_{3}\left(t^{\prime}\right)+2 a_{2}\left(t^{\prime}\right) c_{4}\left(t^{\prime}\right)\right] \exp \left[-c_{5}\left(t^{\prime}\right)\right] \\
& c_{2}(t)=\exp \left[-2 c_{5}(t)\right] \int_{0}^{t} \mathrm{~d} t^{\prime} a_{2}\left(t^{\prime}\right) \exp \left[2 c_{5}\left(t^{\prime}\right)\right] \\
& c_{1}(t)=\int_{0}^{t} \mathrm{~d} t^{\prime}\left[a_{1}\left(t^{\prime}\right)+2 a_{3}\left(t^{\prime}\right) c_{4}\left(t^{\prime}\right)+a_{2}\left(t^{\prime}\right) c_{4}^{2}\left(t^{\prime}\right)\right]
\end{aligned}
$$

Once the coefficients $c_{i}(t)$ are known, the operator $U_{0}(t, 0)$ is uniquely determined.

Next, using the above explicit form of the operator $U_{0}(t, 0)$, we can obtain the exact form of the operator $U_{I}(t)$ :

$$
\begin{aligned}
H_{I}(t)= & \left\{\kappa(t) \theta(t) c_{4}(t)+c_{3}(t) \exp \left[-c_{5}(t)\right]-a_{1}(t)\right\} \frac{\partial}{\partial x} \\
& +\left[\kappa(t) \theta(t)+2 c_{2}(t)\right] \exp \left[c_{5}(t)\right] \frac{\partial}{\partial r}-\exp \left[-c_{5}(t)\right] r
\end{aligned}
$$

It is not difficult to see that the operator $U_{I}(t, 0)$ can be expressed in the form

$$
U_{I}(t, 0)=\exp \left[c_{6}(t) \frac{\partial}{\partial x}\right] \mathcal{U}(t, 0)
$$

where

$$
c_{6}(t)=\int_{0}^{t} \mathrm{~d} t^{\prime}\left\{\kappa\left(t^{\prime}\right) \theta\left(t^{\prime}\right) c_{4}\left(t^{\prime}\right)+c_{3}\left(t^{\prime}\right) \exp \left[-c_{5}\left(t^{\prime}\right)\right]-a_{1}\left(t^{\prime}\right)\right\}
$$

and $\mathcal{U}(t, 0)$ satisfies the evolution equation

$$
\mathcal{H}(t) \mathcal{U}(t, 0) \equiv \sum_{i=1}^{3} b_{i}(t) \hat{e}_{i} \mathcal{U}(t, 0)=\frac{\partial}{\partial t} \mathcal{U}(t, 0), \quad \mathcal{U}(0,0)=1
$$

with

$$
\begin{gathered}
b_{1}(t)=\left[\kappa(t) \theta(t)+2 c_{2}(t)\right] \exp \left[c_{5}(t)\right], \\
b_{2}(t)=-\exp \left[-c_{5}(t)\right], \quad b_{3}(t)=0 \\
\hat{e}_{1}=\frac{\partial}{\partial r}, \quad \hat{e}_{2}=r, \quad \hat{e}_{3}=1
\end{gathered}
$$

The operators $\hat{e}_{i}$ form the Heisenberg-Weyl Lie algebra 


$$
\left[\hat{e}_{1}, \hat{e}_{2}\right]=\hat{e}_{3}, \quad\left[\hat{e}_{1}, \hat{e}_{3}\right]=\left[\hat{e}_{2}, \hat{e}_{3}\right]=0
$$

Following a similar procedure as shown above, the operator $\mathcal{U}(t, 0)$ is found to be

$$
\mathcal{U}(t, 0)=\exp \left[c_{7}(t) \hat{e}_{2}\right] \exp \left[c_{8}(t) \hat{e}_{1}\right] \exp \left[c_{9}(t) \hat{e}_{3}\right]
$$

with

$$
c_{7}(t)=\int_{0}^{t} \mathrm{~d} t^{\prime} b_{2}\left(t^{\prime}\right), \quad c_{8}(t)=\int_{0}^{t} \mathrm{~d} t^{\prime} b_{1}\left(t^{\prime}\right), \quad c_{9}(t)=\int_{0}^{t} \mathrm{~d} t^{\prime} c_{7}\left(t^{\prime}\right) b_{1}\left(t^{\prime}\right)
$$

As a result, we have obtained the exact form of the desired time evolution operator $U(t, 0)$ of the pricing equation in equation (A2).

It is then not difficult to show that $P(x, r, t)$ is given by

$$
P(x, r, t)=\int_{-\infty}^{\infty} \mathrm{d} r^{\prime} \int_{-\infty}^{\infty} \mathrm{d} x^{\prime} G_{0}\left(x, r, t ; x^{\prime}, r^{\prime}, 0\right) P\left(x^{\prime}, r^{\prime}, 0\right)
$$

where

$$
\begin{aligned}
& G_{0}\left(x, r, t ; x^{\prime}, r^{\prime}, 0\right)= \\
& \frac{1}{\sqrt{4 \pi c_{1}(t)}} \cdot \frac{1}{\sqrt{4 \pi c_{2}(t)}} \cdot \frac{1}{\sqrt{1-\eta^{2}(t)}} \exp \left\{\frac{\eta^{2}(t) z_{1}(t) z_{2}(t)}{c_{3}(t)\left[1-\eta^{2}(t)\right]}\right\} \times \\
& \exp \left\{-\frac{z_{1}^{2}(t)}{4 c_{1}(t)\left[1-\eta^{2}(t)\right]}\right\} \exp \left\{-\frac{z_{2}^{2}(t)}{4 c_{2}(t)\left[1-\eta^{2}(t)\right]}\right\} \times \\
& \exp \left\{c_{9}(t)+c_{2}(t) c_{7}^{2}(t) \exp \left[2 c_{5}(t)\right]+c_{7}(t) \exp \left[c_{5}(t)\right] r\right\}
\end{aligned}
$$

is the kernel of the partial differential equation governing the bond price, and

$$
\begin{aligned}
& z_{1}(t)=x^{\prime}-x-c_{4}(t) r-c_{6}(t)-c_{3}(t) c_{7}(t) \\
& z_{2}(t)=r^{\prime}-r \exp \left[c_{5}(t)\right]-c_{8}(t)-2 c_{2}(t) c_{7}(t) \exp \left[2 c_{5}(t)\right] \\
& \eta(t)=\frac{c_{3}(t)}{2 \sqrt{c_{1}(t) c_{2}(t)}}
\end{aligned}
$$

Since $P(x, r, 0)$ is in general independent of $r$ for a bond, Equation (A22) can be reduced to

$$
P(x, r, t)=\int_{-\infty}^{\infty} \mathrm{d} x^{\prime} K_{0}\left(x, t ; x^{\prime}, 0 ; r\right) P\left(x^{\prime}, r, 0\right)
$$

where 


$$
\begin{aligned}
& K_{0}\left(x, t ; x^{\prime}, 0 ; r\right)=\frac{1}{\sqrt{4 \pi c_{1}(t)}} \exp \left[-\frac{z_{1}^{2}(t)}{4 c_{1}(t)}\right] \times \\
& \exp \left\{c_{9}(t)+c_{2}(t) c_{7}^{2}(t) \exp \left[2 c_{5}(t)\right]+c_{7}(t) \exp \left[c_{5}(t)\right] r\right\}
\end{aligned}
$$

We should note that equations (A25) and (A26) represent a very general result for the bond model because the propagator $K_{0}\left(x, t ; x^{\prime}, 0 ; r\right)$ is indeed applicable for any arbitrary $P(x, r, 0)$, which depends on $\mathrm{x}$ only. Furthermore, it is easy to show that in terms of the riskless bond function $Q(r, t)$ of the Vasicek model with explicitly time-dependent parameters, both $z_{1}(t)$ and $K_{0}\left(x, t ; x^{\prime}, 0 ; r\right)$ can be rewritten as follows:

$$
\begin{aligned}
z_{1}(t) & =x^{\prime}-x+\ln [Q(r, t)]+c_{1}(t) \\
K_{0}\left(x, t ; x^{\prime}, 0 ; r\right) & =\frac{Q(r, t)}{\sqrt{4 \pi c_{1}(t)}} \exp \left[-\frac{z_{1}^{2}(t)}{4 c_{1}(t)}\right]
\end{aligned}
$$

Finally, using an approach based upon the method of images, we can straightforwardly incorporate an absorbing barrier with a drifted dynamics of the form $H(r, t)=X Q(r, t) \exp \left[\beta c_{1}(t)\right]$ into our model, where $X$ is the pre-defined asset value of the barrier and $\beta$ is a real adjustable parameter. The corresponding bond price $P(y, r, t)$ is then given by

$$
\begin{aligned}
P(y, r, t) & =\exp \left(-\frac{\beta-1}{2} y\right) \int_{0}^{\infty} \mathrm{d} y^{\prime} G\left(y, t ; y^{\prime}, 0 ; r\right) \\
& \times \exp \left(\frac{\beta-1}{2} y^{\prime}\right) P\left(y^{\prime}, r, 0\right) \\
G\left(y, t ; y^{\prime}, 0 ; r\right) & =K\left(y, t ; y^{\prime}, 0 ; r\right)-K\left(y, t ;-y^{\prime}, 0 ; r\right) \\
K\left(y, t ; y^{\prime}, 0 ; r\right) & =\frac{Q(r, t)^{(\beta+1) / 2} \exp \left[\left(\beta^{2}-1\right) c_{1}(t) / 4\right]}{\sqrt{4 \pi c_{1}(t)}} \\
& \times \exp \left(-\frac{\left\{y^{\prime}-y+\ln [Q(r, t)]+\beta c_{1}(t)\right\}^{2}}{4 c_{1}(t)}\right)
\end{aligned}
$$

where $y=\ln (A / X)$ and $y^{\prime}=\ln \left(A^{\prime} / X\right)$. It should be noted that this solution vanishes at the barrier; that is, it is the solution associated with the homogeneous boundary condition only. Nevertheless, it is an easy task to extend the solution to satisfy the inhomogeneous boundary condition: $P(A, r, t)=\alpha_{1} X Q(r, t)$ at $A=$ $H(r, t)$, by simply adding the trivial solution $\alpha_{1} X Q(r, t)$ of the pricing equation in equation (A1). Then, by requiring that the solution associated with the inhomogeneous boundary condition obeys the final payoff condition in equation (8), 
we can readily obtain the desired corporate bond function of our model in equation (9).

1. Pricing corporate bonds without any default barrier based on stochastic interest rates following the Ornstein-Uhlenbeck process is studied by Shimko et al (1993).

2. Schöbel (1999) develops a model incorporating the same dynamics for the default barrier as that in the BV model. The default barrier takes the form of a fraction of risk-free bond price with the face value of the corporate bond.

3. It will be shown in the next section that the parameter $\beta$ and the variance of the corporate bond value can be combined as a single parameter to adjust the dynamics of the default barrier.

4. Although this assumed process is consistent with many of the observed properties of interest rates, it can allow negative interest rates. However, this assumption may still be justifiable in the context of the valuation because the probability of negative interest rates occurring is small for realistic parameters. In addition, given that the current value of interest rate and the mean-level are both positive, the dynamics always imply positive expected future interest rate.

5. Campbell (1986) shows that a constant market price of risk $\lambda$ can be justified in a market equilibrium with log-utility investors.

6. It can be shown by completing square of $c_{1}(t)$.

7. Given a positive $\beta$ and $\alpha_{1} \leq 1$, the payoff upon default is less than or equal to the firm value as $P(A=H, r, t)=a_{1} X Q(r, t) \leq A=X Q(r, t) \exp \left[\beta c_{1}(t)\right]$.

8. Johnson observes that low quality bonds have very high credit spreads at short maturities. He explains this phenomenon by arguing that default probabilities are high near maturity, because there is uncertainty about the ability to refinance the bond. This explanation assumes, as Johnson points out, that firms are unable to accumulate cash before maturity for debt repayment before maturity.

\section{REFERENCES}

Altman, E. I. (1989). Measuring corporate bond mortality and performance. Journal of Finance 44, 909-22.

Black, F., and Scholes, M. (1973). The pricing of options and corporate liability. Journal of Political Economics 81, 637-54.

Black, F., and Cox, J. (1976). Valuing corporate securities: some effects of bond indenture provisions. Journal of Finance 35, 1223-34.

Briys, E., and de Varenne, F. (1997). Valuing risky fixed rate debt: an extension. Journal of Financial and Quantitative Analysis 32, 230-48.

Campbell J. Y. (1986). A defence of traditional hypotheses about the term structure of interest rates. Journal of Finance 41, 183-93.

Carty, L. V. and Lieberman, D. (1998). Historical default rates of corporate bond issuers, 1920-1996. in Satyajit Das ed.: Credit Derivatives (John Wiley, Singapore).

Fama, E. F. (1986). Term premiums and default premiums in money markets. Journal of Financial Economics 17, 175-96.

Fons, J. S. (1986). Using default rates to model the term structure of credit risk. Financial Analysts Journal September-October, 25-32.

Johnson, R. E. (1967). Term structures of corporate bond yields as a function of risk of default. Journal of Finance 22, 313-45. 
Longstaff, F. and E. Schwartz. (1995). A simple approach to valuing risky fixed and floating rate debt. Journal of Finance 50 , 789-819.

Madan, D. B., and Unal, H. (2000). A two-factor hazard-rate model for pricing risky debt and the term structure of credit spreads. Journal of Financial and Quantitative Analysis 35, $43-65$.

Merton, R. C. (1974). On the pricing of corporate debt: the risk structure of interest rates. Journal of Finance 2, 449-70.

Modigliani, F., and Miller, M. (1958). The cost of capital, corporate finance, and the theory of investment. American Economics Review 48, 261-97.

Sarig, O., and Warga, A. (1989). Some empirical estimates of the risk structure of interest rates. Journal of Finance 44, 1351-60.

Schöbel, F. (1999). A note on the valuation of risky corporate bonds. OR Spektrum, 21, 35-47.

Shimko, D. C., Tejima, N., and Van Deventer, D. R. (1993). The pricing of risky debt when interest rates are stochastic. Journal of Fixed Income 3, 58-65.

Standard and Poor's (2001). Adjusted key U.S. industrial financial ratios.

Vasicek, O. A. (1977). An equilibrium characterisation of the term structure. Journal of Financial Economics 5, 177-88.

Wei, J., and Norman, E. (1963). Lie algebraic solution of linear differential equations. Journal of Mathematical Physics 4, 575-81.

Zhou, C. (1997). A jump-diffusion approach to modeling credit risk and valuing defaultable securities. Working Paper, Board of Governors of the Federal Reserve System. 
